\title{
IMMUNOHISTOCHEMICAL STUDY OF SYNDECAN-1 EXPRESSION IN DIFFERENT HISTOLOGICAL SUBTYPES OF AMELOBLASTOMA AND ODONTOGENIC KERATOCYSTS
}

\author{
Urvashi A. Shetty1, Chethana Dinakar², Pushparaja Shetty33, Kumuda Rao ${ }^{4}$ Reshma Amin ${ }^{5}$
}

${ }_{1}^{1}$ Senior Lecturer, Department of Oral Pathology and Microbiology, A. B. Shetty Memorial Institute of Dental Sciences, NITTE Deemed to be University, Deralakatte, Mangalore, Karnataka, India.

${ }^{2}$ Reader, Department of Oral Pathology and Microbiology, A. B. Shetty Memorial Institute of Dental Sciences, NITTE Deemed to be University, Deralakatte, Mangalore, Karnataka, India.

3Professor and HOD, Department of Oral Pathology and Microbiology, A. B. Shetty Memorial Institute of Dental Sciences, NITTE

Deemed to be University, Deralakatte, Mangalore, Karnataka, India.

${ }^{4}$ Senior Lecturer, Department of Oral Medicine and Radiology, A. B. Shetty Memorial Institute of Dental Sciences, NITTE Deemed to be

University, Deralakatte, Mangalore, Karnataka, India.

${ }^{5}$ Senior Lecturer, Department of Oral Pathology and Microbiology, A. B. Shetty Memorial Institute of Dental Sciences, NITTE Deemed to be University, Deralakatte, Mangalore, Karnataka, India.

\section{BACKGROUND}

ABSTRACT

Assessment of cell proliferation activity in tumours has emerged as a common tool used by histopathologists to gain useful information for predicting the behaviour of tumours and their likelihood of local recurrence, metastatic potential, the duration of disease-free survival. It is important to demonstrate specific and sensitive techniques for determining the presence of these proliferation markers.

Aims and Objectives-The purpose of this study was to evaluate the intensity, localization, and distribution of Syndecan expression in Ameloblastomas and Odontogenic Keratocysts (OKC).

\section{MATERIALS AND METHODS}

Total of 40 cases (20 each) of Ameloblastoma (8 Unicystic ameloblastoma \& 12 Solid/multicystic ameloblastoma) and OKC was taken, and immunohistochemical staining was performed using the primary antibody for syndecan using two -step detection system based on HRP labelled polymer technique. Sample size taken for convenience. Comparison of intensity, localization, and distribution of Syndecan (CD138) between Ameloblastoma and OKC was analysed using the chi-square test/ Fishers exact test. P value of $<0.05$ was considered as statistically significant. Interobserver agreement was done using Kappa statics which showed score of 0.88 suggesting good agreement.

\section{RESULTS}

All 40 cases (20 each) of Ameloblastoma and OKC showed positivity for syndecan (CD138). OKC showed more diffuse distribution and intense staining when compared to Ameloblastoma.

\section{CONCLUSION}

The histologic presentation of ameloblastoma, especially unicystic type, can be, in some instances, mistaken for odontogenic keratocyst (OKC) because of their overlapping clinical and radiographic presentation. The expression of syndecan-1 in ameloblastoma showed reduced immunostaining of tumour epithelium when compared to OKC. It was also noted that the decreased expression of syndecan-1 in SMA supports the view that this variant of ameloblastoma has an aggressive biological behaviour than the UA.

\section{KEY WORDS}

Immunohistochemistry, Syndecan, Ameloblastoma, Tumour Marker, Odontogenic Keratocyst.

HOW TO CITE THIS ARTICLE: Shetty UA, Dinakar C, Shetty P, et al. Immunohistochemical study of syndecan-1 expression in different histological subtypes of ameloblastoma and odontogenic keratocysts. J. Evolution Med. Dent. Sci. 2019;8(04):260-264, DOI: $10.14260 /$ jemds/2019/56

\section{BACKGROUND}

The practice of Oral pathology is currently undergoing significant change with advances in the field of molecular and diagnostic techniques, one of which is the use of tumour

'Financial or Other Competing Interest': None.

Submission 24-10-2018, Peer Review 15-01-2019,

Acceptance 22-01-2019, Published 28-01-2019.

Corresponding Author:

Dr. Chethana Dinakar,

Reader, Department of Oral Pathology and Microbiology,

A. B. Shetty Memorial Institute of Dental Sciences, Nitte Deemed to be

University, Deralakatte, Mangalore-575018, Karnataka, India.

E-mail:dr.chethana23@gmail.com

DOI: $10.14260 /$ jemds $/ 2019 / 56$

\section{(c) (i) $(-)$}

markers. Tumour markers help Oral pathologists for confirmatory diagnosis of histopathologically confounding lesions. ${ }^{1}$

Tumour markers are categorised into prognostic and diagnostic markers. Specific diagnostic markers appear significantly in cells of a particular neoplasm. These markers can be used to assess the histogenic origin and cellular lineage of various neoplasms. Even the most perplexing tumours can be distinguished with the help of specific diagnostic markers. On the other hand, prognostic markers can determine the growth, invasion and metastasis potential of tumours. ${ }^{2}$

Syndecan-l (SDC 1) is also known as CD 138 is a transmembrane heparan sulfate. It has a role in the regulation of cellular differentiation, morphology and adhesion, hence, 
loss of which can be associated with decreased adhesion, disordered differentiation and uncontrolled proliferation of tumour cells. ${ }^{3}$ A cell's potential invasive behaviour has been linked to the down-regulation of syndecan -1.4

Odontogenic tumours like Ameloblastoma and Odontogenic Keratocyst (OKC) can pose diagnostic challenges because of their overlapping clinical, radiographic and histopathological presentation. Tumour markers can be helpful for accurate diagnosis as their prognosis and treatment modality differ but lack of comprehensive literature on diagnostic markers for odontogenic tumours has hindered the process. Even though histopathology continues to be the staple in the diagnosis of odontogenic tumours, tumour markers can play an increasingly important role as adjuvant diagnostic tools. Also, identification of prognostic tumour markers may be helpful to predict their biological behaviour to assess their proliferation, invasiveness \& recurrence rates.

There are only a handful of tumour markers that can be used by pathologists for diagnosis of odontogenic tumours, and many other potential markers are constantly under development. Hence, this study was designed to evaluate the intensity, localization, and distribution of Syndecan expression in Ameloblastomas and Odontogenic Keratocysts (OKC).

\section{MATERIALS AND METHODS}

An observational study was done on 20 cases of Ameloblastoma (Unicystic and multicystic variants) and 20 cases of Odontogenic Keratocyst taken from archives of the Department. Re-evaluation of all cases was performed according to 2017 WHO classification of odontogenic tumours and cysts by two oral pathologists on light microscopy using Haematoxylin and Eosin sections. Sample size taken for convenience

The study protocol was reviewed by the Ethical Committee of our University and was granted ethical clearance. Cases not satisfying the histopathological criteria outlined by the 2017 World Health Organization classification of Odontogenic Tumours, Hybrid Odontogenic Tumours/ Collision tumour, OKC associated with Nevoid Basal cell syndrome were grouped under exclusion criteria.

Formalin-fixed paraffin-embedded tissue blocks were cut into $4 \mu \mathrm{m}$ thick sections and placed on positive charged Autofrost slides. Immunohistochemical staining procedure was performed using the primary antibody for Syndecan-1 using two-step detection system based on -HRP labelled polymer technique (Path $\mathrm{n}$ situ Biotechnologies) according to the manufacturer instructions.

Evaluation of the stained slides was carried out by two pathologists for the distribution, presence, and intensity of the immunoreactive cells using a binocular research microscope (Lawrence \& Mayo) under $100 \mathrm{x}$ and 400x magnification. Presence was evaluated to estimate whether the staining was positive or negative and, if positive, which epithelial layer was stained. Distribution was evaluated as being either focal (involving $<50 \%$ of positive cells) or diffuse (involving $>50 \%$ of positive cells). The intensity was graded based on the number of positive cells seen: $0=$ no staining; $1=$ weak staining; 2 = moderate staining; 3 = intense staining.

\section{Statistical Analysis}

The data collected was entered into Microsoft excel spread sheet and analysed using IBM SPSS Statistics, Version 22 (Armonk, NY: IBM Corp). Comparison of intensity, localization, and distribution of Syndecan (CD138) in Ameloblastoma and OKC was analysed using chi-square test/Fishers exact test. P value of $<0.05$ was considered as statistically significant. Inter observer agreement was done using Kappa statics which showed sore of 0.88 suggesting good agreement.

\section{RESULTS}

A total of 40 cases (20 each) of Ameloblastoma and OKC were studied. Out of the 20 cases of Ameloblastoma, 8 were of Unicystic type, and the remaining 12 were of Solid/multicystic type [4 Follicular, 4 Plexiform, 2 Acanthomatous, 1 Granular, 1 Desmoplastic type of Ameloblastoma.

\section{Expression of Syndecan in Ameloblastoma and OKC}

All the 20 cases of ameloblastoma \& 20 cases of OKC showed positivity to Syndecan. In all the 20 immune positive cases of Ameloblastoma, 17 (85\%) showed reactivity of Syndecan towards cell membrane as well as cytoplasm. Only $3(15 \%)$ showed positivity to cell membrane, cytoplasm, and nucleus in focal areas (Figure 1, 1a, 1b) whereas in cases of OKC, $12(60.0 \%)$ showed reactivity in the cell membrane and cytoplasm and $8(40 \%)$ cases showed reactivity in the cell membrane, cytoplasm, and nucleus. The difference of which was statistically significant (Table 1).

Diffuse distribution of Syndecan was seen more in OKC [14 (70\%)] when compared to Ameloblastoma which showed positivity in only $9(45 \%)$ cases. Focal distribution was more seen in Ameloblastoma 11 (55\%) when compared with OKC which showed positivity in only $6(30 \%)$ of cases. (Table 2 ) Most of the Ameloblastoma cases [15 (75\%)] showed moderate and weak staining intensity when compared to OKC which showed moderate and strong staining intensity [2 $(10 \%)]$. Comparison of the intensity of syndecan stain between ameloblastoma and OKC shows that there was a significant difference among the various staining intensities for both lesions (Table 3)

In the cystic lining of OKC, syndecan-1 was highly expressed on the cell surfaces of the keratinocytes in $92.3 \%$ of the cases. There was no syndecan-1 expression in the parakeratin layer. However, it was not uniformly expressed with the presence of some negative focal areas (Figure 2, 2a). Some cases showed satellite cysts and budding of the basal layer into the connective tissue. The satellite cysts did not differ from their main cysts in the expression of syndecan-1, (Figure 2b). while the epithelial budding showed decreased syndecan-1 expression. 


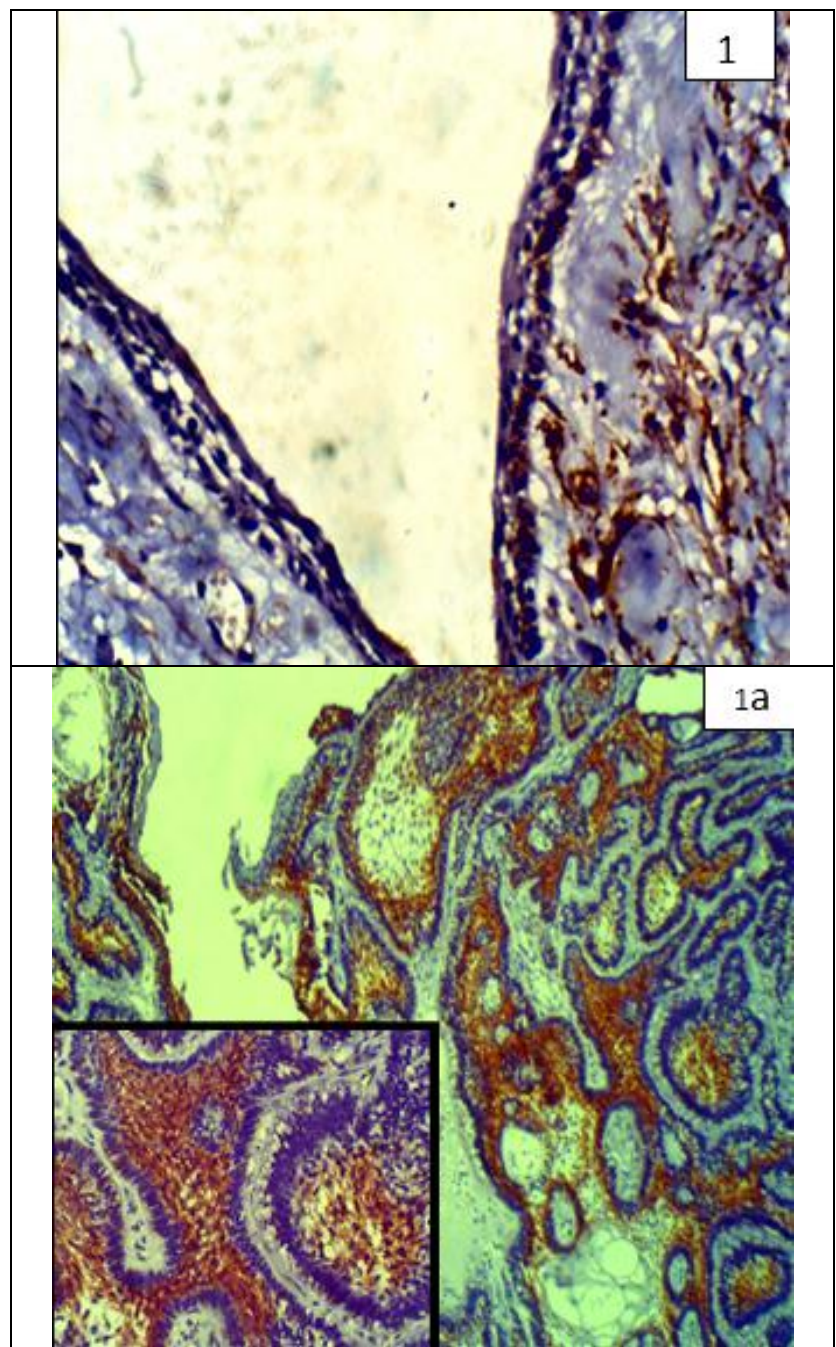

Figure 1, 1a: Photomicrograph Showing Syndecan-1 Expression in Unicystic Ameloblastoma (400x) \& Plexiform Ameloblastoma (100x) And 400x (Inset)

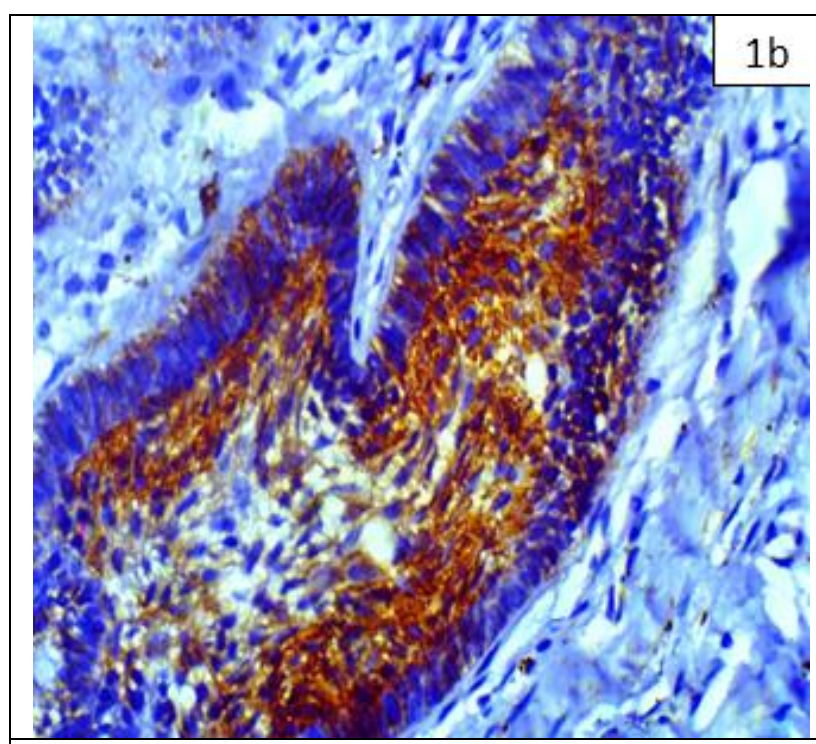

Figure 1b. Photomicrograph showing Syndecan-1 expression in follicular ameloblastoma. The central cells were showing higher expression than the peripheral cells.

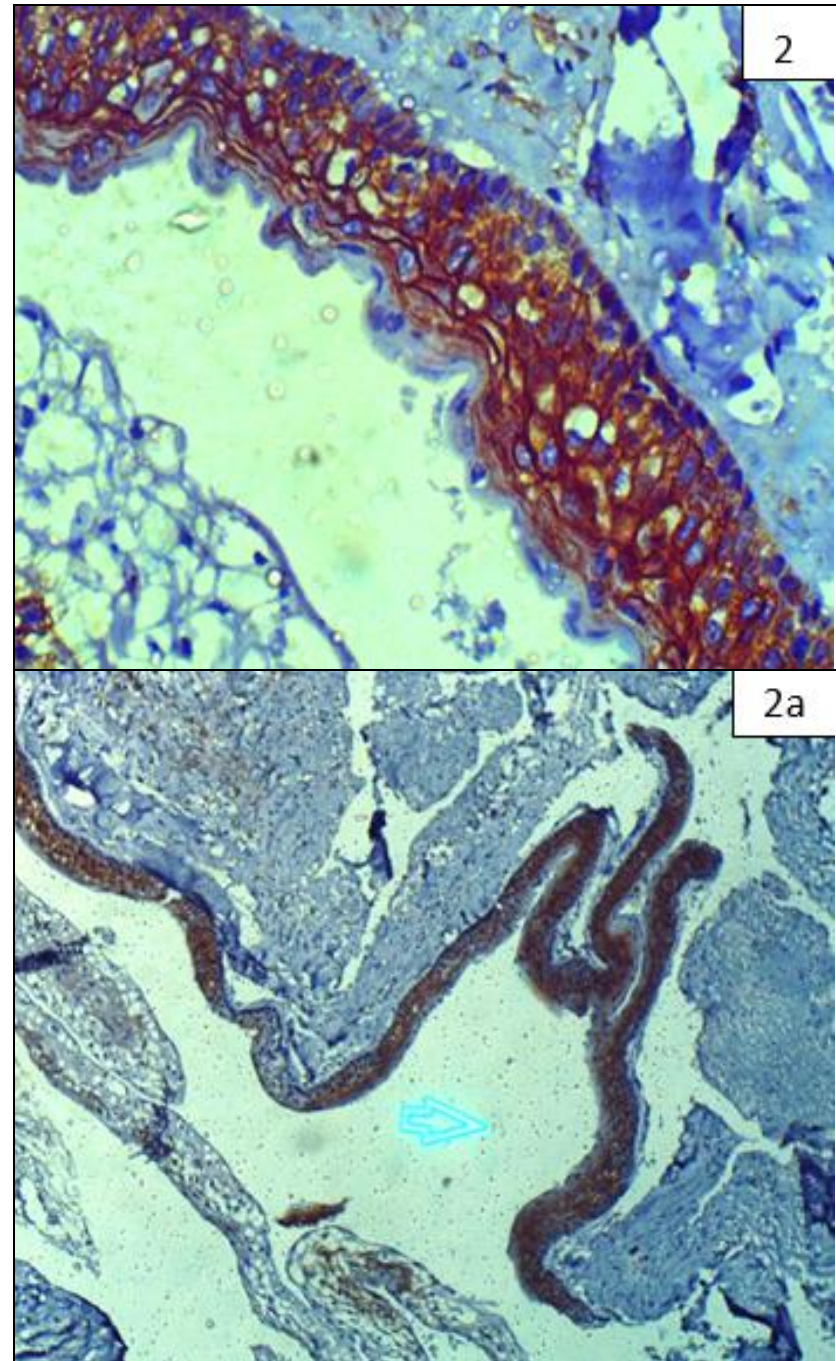

Figure: 2, 2a: Photomicrograph Showing Syndecan-1 Expression in OKC $(400 x \& 40 x)$

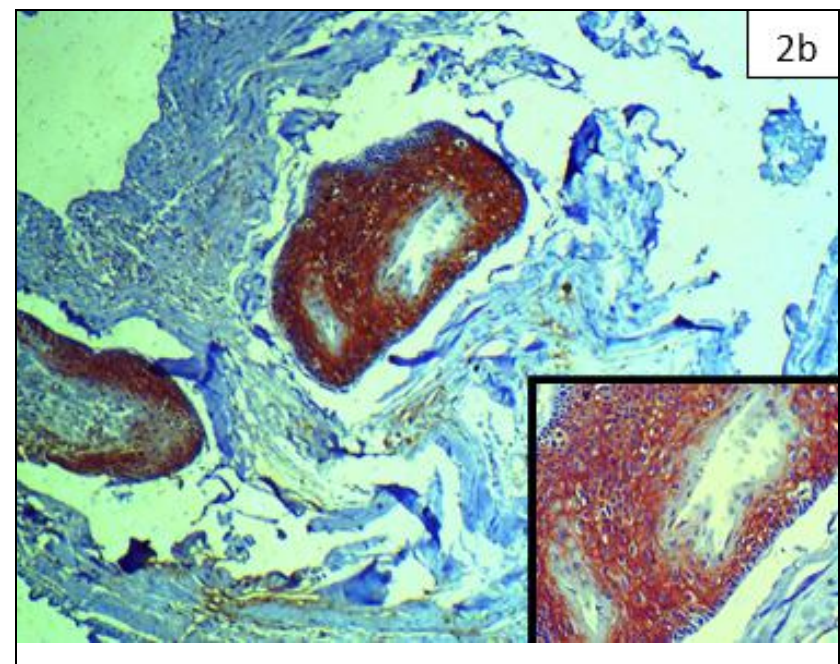

Figure 2b: Photomicrograph Showing Syndecan-1 Expression in Daughter Cyst of OKC (40x) and 100x (Inset) (400x). 


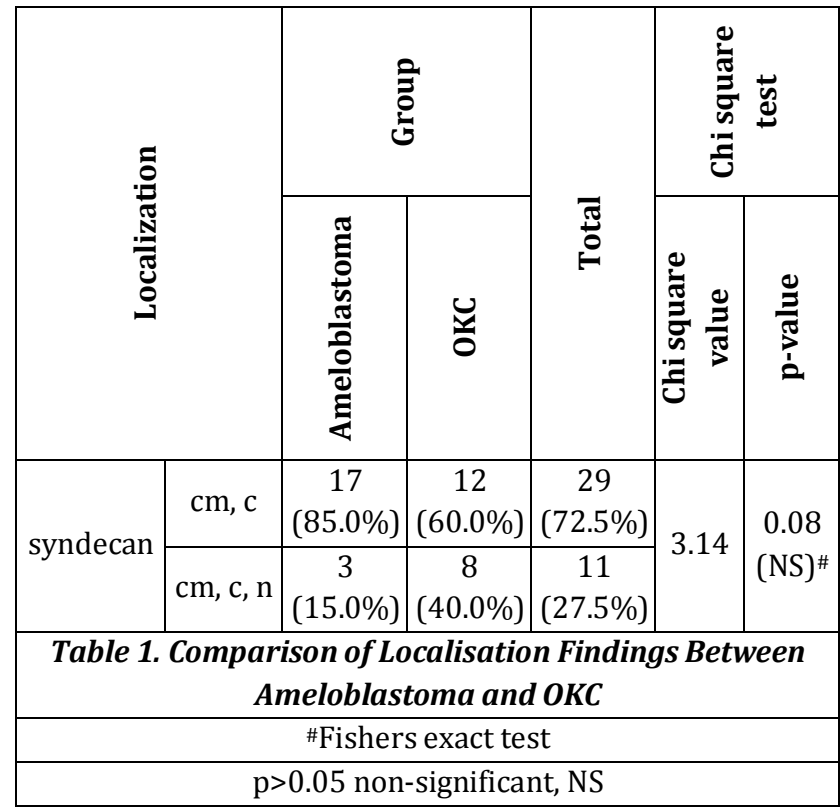

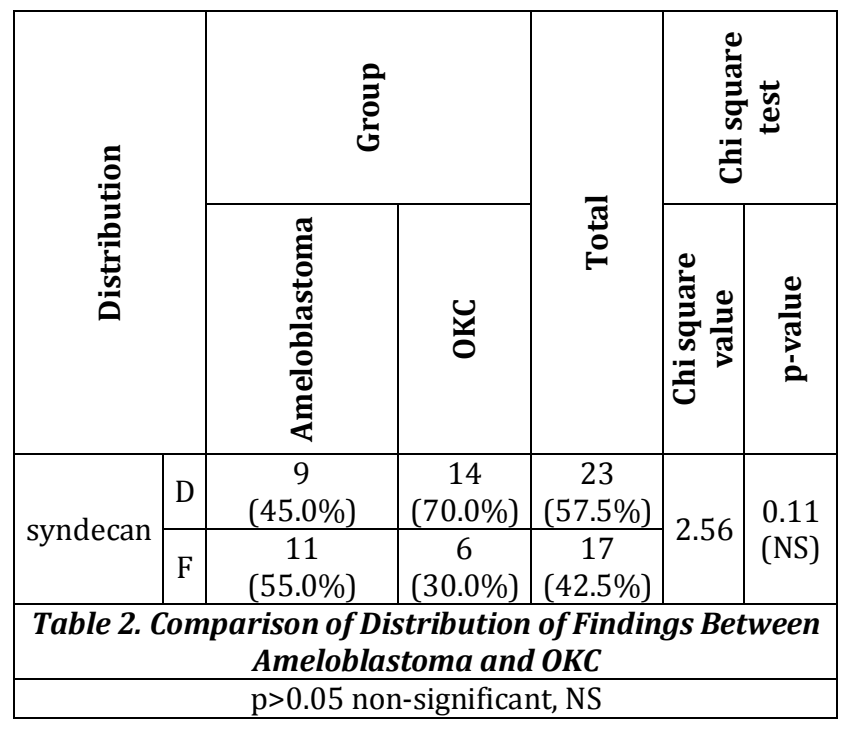

\begin{tabular}{|c|c|c|c|c|c|c|}
\hline \multirow{2}{*}{ 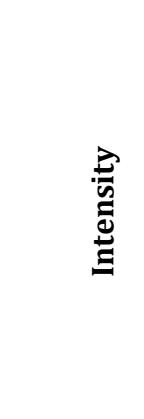 } & & \multicolumn{2}{|c|}{$\begin{array}{l}\hat{Z} \\
\text { ㅎํㄴ }\end{array}$} & \multirow[b]{2}{*}{ 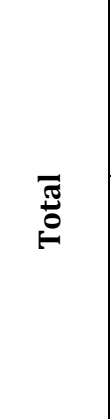 } & \multicolumn{2}{|c|}{ 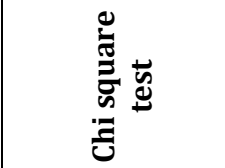 } \\
\hline & & 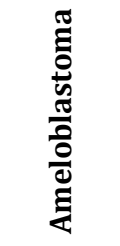 & 幽 & & & 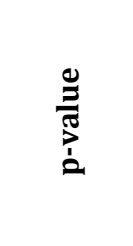 \\
\hline \multirow{3}{*}{ syndecan } & 1 & 0 & 0 & 0 & \multirow{3}{*}{17.29} & \multirow{3}{*}{$<0.001^{\text {*\# }}$} \\
\hline & 2 & $\begin{array}{c}15 \\
(75.0 \%)\end{array}$ & $\begin{array}{c}2 \\
(10.0 \%)\end{array}$ & $\begin{array}{c}17 \\
(42.5 \%)\end{array}$ & & \\
\hline & 3 & $\begin{array}{c}5 \\
(25.0 \%)\end{array}$ & $\begin{array}{c}18 \\
(90.0 \%)\end{array}$ & $\begin{array}{c}23 \\
(57.5 \%)\end{array}$ & & \\
\hline \multicolumn{7}{|c|}{$\begin{array}{l}\text { Table 3. Comparison of Intensity Between } \\
\text { Ameloblastoma and OKC }\end{array}$} \\
\hline \multicolumn{7}{|c|}{ \#Fishers exact test } \\
\hline${ }^{*} \mathrm{p}<0.05$ & & y sign & ificant & 0.05 & 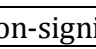 & \\
\hline
\end{tabular}

\section{DISCUSSION}

Odontogenic cysts and tumours are lesions arising from the odontogenic apparatus, from the odontogenic epithelium and/or ectomesenchyme with varying degrees of inductive tissue interaction. ${ }^{5}$ Their inconstant appearance may lead to problems in diagnosis with infrequent serious confusion with more menacing lesions. ${ }^{6}$

Among the epithelial odontogenic tumours, Ameloblastoma is a locally aggressive, benign odontogenic tumour with a potential to become malignant and produce distant metastasis to kidneys and lungs. ${ }^{7}$ They have been grouped broadly into three biologic variants: cystic (Unicystic), solid, and peripheral. The cystic variant is biologically less aggressive and responds better to enucleation or curettage than the solid Ameloblastoma. 8 The clinical course of ameloblastoma is difficult to predict because there are few features to evaluate the biological behaviour of the tumour, ${ }^{9}$ on the other hand OKC is an aggressive cyst with neoplastic behaviour which also originates from cell rests of the dental lamina, same as ameloblastoma, and there are radiographic and clinical similarities between OKC and unicystic ameloblastoma, as both present as ordinary cysts in the dentate areas. ${ }^{10}$

Most of the times, histologically, SMA \& unicystic ameloblastoma is lined most commonly, by the odontogenic epithelium and stratified squamous epithelium in the remaining areas. ${ }^{8}$ Unicystic ameloblastoma may present with squamous metaplasia frequently, and sometimes by a nondescript epithelium, which can create diagnostic confusion with odontogenic cysts. ${ }^{11} \mathrm{OKC}$ is characterized, histologically, by a palisaded basal cell layer of basophilic columnar cells and a surface of corrugated parakeratin, sometimes with spongiosis, resembling closely the stellate like reticulum and the acanthomatous differentiation of Ameloblastoma. If the neoplastic epithelium displays reactive changes induced by inflammation, it can mimic unicystic ameloblastoma histologically. 12

There is no clear evidence that histologic variants of ameloblastoma, such as follicular/plexiform/unicystic patterns, regulate the degree of invasiveness of individual cases or allow prediction of the likelihood of recurrence or malignant transformation. Thus, at times, both lesions become histologically indistinguishable. So, the use of a predictive marker might help to narrow down the diagnostic confusion. In the present study, two major odontogenic lesions were chosen for immunohistochemical evaluation, due to their common occurrence among other odontogenic lesions and their interesting clinical and biological behaviour. ${ }^{10}$ Other odontogenic tumours were not analysed in this study because of the inadequate number of available cases. Inflammatory odontogenic cysts were not included in this study, to avoid its attributed inflammatory effect on the expression of syndecan1.

The present study assessed the expression of Syndecan -1 in 20 cases each of various subtypes of ameloblastoma and odontogenic keratocysts.

There is a need for a predictive marker of clinical behaviour in ameloblastoma subtypes \& OKC. The proliferative activity of tumour cells has been proven to be an indicator of aggressiveness and to give useful prognostic information.

Specific interactions between cell surfaces and extracellular matrix (ECM) molecules act as the basis of tissue integrity and function. Domains on the extracellular matrix can 
provide attachment sites for cell surface molecules and are hence called matrix receptors. ${ }^{13}$

The differential expression of SD-1 in ameloblastoma could be a reflection of cell type and characteristics, primarily dictated by stages of differentiation and cellular kinetic parameters. Leocata et al ${ }^{14}$ noted that syndecan- 1 is expressed in ameloblastomas as well as in normal epithelial cells and during odontogenesis. Both Ameloblastoma \& OKC are locally invasive neoplasms with a relatively high local recurrence rate.

Cellular invasion necessitates the breakdown of basement membrane and surrounding ECM followed by the proliferation of cells. Therefore, reduced intercellular adhesion and changes in basement membrane composition influence the growth of neoplasms. ${ }^{15}$ According to Leocata et al. ${ }^{14}$ expression of syndecan-1 in ameloblastoma is reduced in relation to normal cells of tooth buds. In the present study, we evaluated the expression of syndecan-1 in ameloblastoma which showed reduced immunostaining of tumour epithelium when compared to OKC. In the present study, it was noticed that the decreased expression of syndecan-1 in SMA supports the view that this variant of ameloblastoma has an aggressive biological behaviour than the UA. Reduced syndecan-1 expression is assumed to have a major role in the invasive and infiltrative potentials of tumour islands in the surrounding stroma.

\section{CONCLUSION}

The histologic presentation of ameloblastoma, especially unicystic type, can be, in some instances, mistaken for odontogenic keratocyst (OKC) because of their overlapping clinical and radiographic presentation.

Preoperative immunohistochemistry analysis may be considered as an important step for the treatment plan. Hence, within the framework of the present study, the following conclusions and recommendations seem appropriate:

The early detection of the high proliferative activity and aggressive characteristics of such lesions may give a possible molecular basis for their clinical behaviour which may have a significant impact in their prognosis.

Molecular and biochemical studies are required to determine the detailed mechanism of syndecan-1 and their related molecules in odontogenic lesions which may lead to the development of new diagnostic and therapeutic applications.

\section{REFERENCES}

[1] Premalatha BR, Patil S, Rao RS, et al. Odontogenic tumour markers - an overview. J Int Oral Health 2013;5(2):59-69.
[2] Varghese SS, Mathew P, Jose J. Diagnostically relevant molecular markers in head and neck neoplasms. Article ID 873953, ISRN Biomarkers 2013;2013:1-6.

[3] Liu W, Litwack ED, Stanley MJ, et al. Heparan sulfate proteoglycans as adhesive and anti-invasive molecules. Syndecans and glypican have distinct functions. J Biol Chem 1998;273(35):22825-32.

[4] Soukka T, Pohjola J, Inki P, et al. Reduction of syndecan1 expression is associated with dysplastic oral epithelium. J Oral Pathol Med 2000;29(7):308-13.

[5] Fernandes AM, Duarte EC, Pimenta FJ, et al. Odontogenic tumours: a study of 340 cases in a Brazilian population. Journal of Oral Pathology and Medicine 2005;34(10):583-7.

[6] Jordan RCK, Speight PM. Current concepts of odontogenic tumours. Diagnostic Histopathology 2009;15(6):303-10.

[7] Chitra A, Rashmi M, Karanprakash S. Calretinin as a diagnostic adjunct for ameloblastoma. Article ID 308240. Patholog Res Int 2014;2014:1-7.

[8] Philipsen HP, Reichart PA. Unicystic ameloblastoma. A review of 193 cases from the literature. Oral Oncology 1998;34(5):317-25.

[9] Robinson L, Martinez MG. Unicystic ameloblastoma: a prognostically distinct entity. Cancer 1977;40(5):227885.

[10] Neville BW, Damn DD, Allen CM, et al. Oral and maxillofacial pathology. Philadelphia, PA, USA: WB Saunders 2002.

[11] Coleman H, Altini M, Ali $\mathrm{H}$, et al. Use of calretinin in the differential diagnosis of unicystic ameloblastomas. Histopathology 2001;38(4):312-7.

[12] DeVilliers P, Liu H, Suggs C, et al. Calretinin expression in the differential diagnosis of human ameloblastoma and keratocystic odontogenic tumour. The American Journal of Surgical Pathology 2008;32(2):256-60.

[13] Buck CA, Horwitz AF. Cell surface receptors for extracellular matrix molecules. Annu Rev Cell Biol 1987;3:179-205.

[14] Leocata P, Villari D, Fazzari C, et al. Syndecan-1 and Wingless-type protein-1 in human ameloblastomas. J Oral Pathol Med 2007;36(7):394-9.

[15] Kurokawa H, Matsumoto S, Murata T, et al. Immunohistochemical study of syndecan-1 downregulation and the expression of p53 protein or Ki- 67 antigen in oral leukoplakia with or without epithelial dysplasia. J Oral Pathol Med 2003;32(9):513-21. 